\title{
First-Principles Modeling of Vibrational Electron Energy Loss Spectra
}

\author{
G Radtke $^{1 *}$, D Taverna ${ }^{1}$, M Lazzeri $^{1}$, E Balan $^{1}$, FS Hage $^{2}$, QM Ramasse $^{2,3,4}$, OL Krivanek $^{5}$ and TC \\ Lovejoy $^{5}$ \\ 1. Institut de Minéralogie, de Physique des Matériaux, et de Cosmochimie (IMPMC), Sorbonne \\ Université, Muséum National d'Histoire Naturelle,Paris, France. \\ 2. SuperSTEM Laboratory, SciTech Daresbury, Keckwick Lane, Daresbury, U.K. \\ 3. School of Physics, University of Leeds, Leeds, U.K. \\ 4. School of Chemical and Process Engineering, University of Leeds, Leeds, U.K. \\ 5. Nion Co., Kirkland WA, USA. \\ * Corresponding author: guillaume.radtke@sorbonne-universite.fr
}

Probing low energy excitations with electron energy loss spectroscopy (EELS) in the transmission electron microscope has long been hindered by the substantial energy spread of the primary electron beam. Impressive instrumental developments, however, leading to an improvement of the energy resolution down to less than $10 \mathrm{meV}$ and to a drastic reduction of the tail of the zero-loss peak, demonstrated recently the capability of EELS to detect well-defined vibrational excitations in the infrared range of frequencies with an unprecedented spatial resolution $[1,2]$.

Most of the systems of interest in materials, earth or life sciences, however, are built from complex molecules or crystals with many atoms per unit cells giving rise to a large number of closely spaced vibrational modes. A straightforward interpretation of the resulting spectra based on empirical models or on a simple comparison with known references is thus often incomplete and even misleading. A proper assessment of the origin of the different structures observed experimentally therefore relies both on firstprinciple calculations and on an accurate description of the scattering process [3].

In this talk, I will give a general overview of the methods which can be employed to model vibrational EELS spectra on the basis of density functional calculations. In particular, I will present results obtained in near-field geometry, corresponding to a regime dominated by long-range dipole scattering, and on different types of materials including molecular crystals and boron-based super-hard compounds. Figure 1 illustrates very recent experimental results obtained on crystalized $\beta$-guanine. The spectrum has been acquired with an energy resolution of $5.9 \mathrm{meV}$ (2000 spectra of $100 \mathrm{~ms}$ acquisition time each) at $30 \mathrm{keV}$ acceleration voltage and about $50 \mathrm{~nm}$ away from the crystal surface. All the EELS active modes between 300 and $3500 \mathrm{~cm}^{-1}$ are visible, extending considerably the frequency range accessible three years ago $[3,4]$. As it will be discussed, the qualitative agreement obtained with state-of-the-art density functional calculations [3] (see Figure 1) allows an unambiguous interpretation of the origin of most of the visible structures. Besides bond stretching modes located at high energies, the spectrum now indeed reveals a series of intense peaks associated with lower-energy in-plane and out-of-plane bond bending modes.

Finally, I will give a brief presentation of the formalism employed when modelling experiments carried out in transmission geometry, a regime dominated by impact scattering, where a sizeable momentum is transferred to the specimen and how it can be implemented in the framework of density functional theory calculations. 
References:

[1] OL Krivanek et al., Nature 514 (2014), p. 209.

[2] MJ Lagos et al., Nature 543 (2017), p. 529.

[3] G Radtke et al., Phys. Rev. Lett. 119 (2017), p. 027402.

[4] P Rez et al., Nature Comm. 7 (2016), p. 10945.

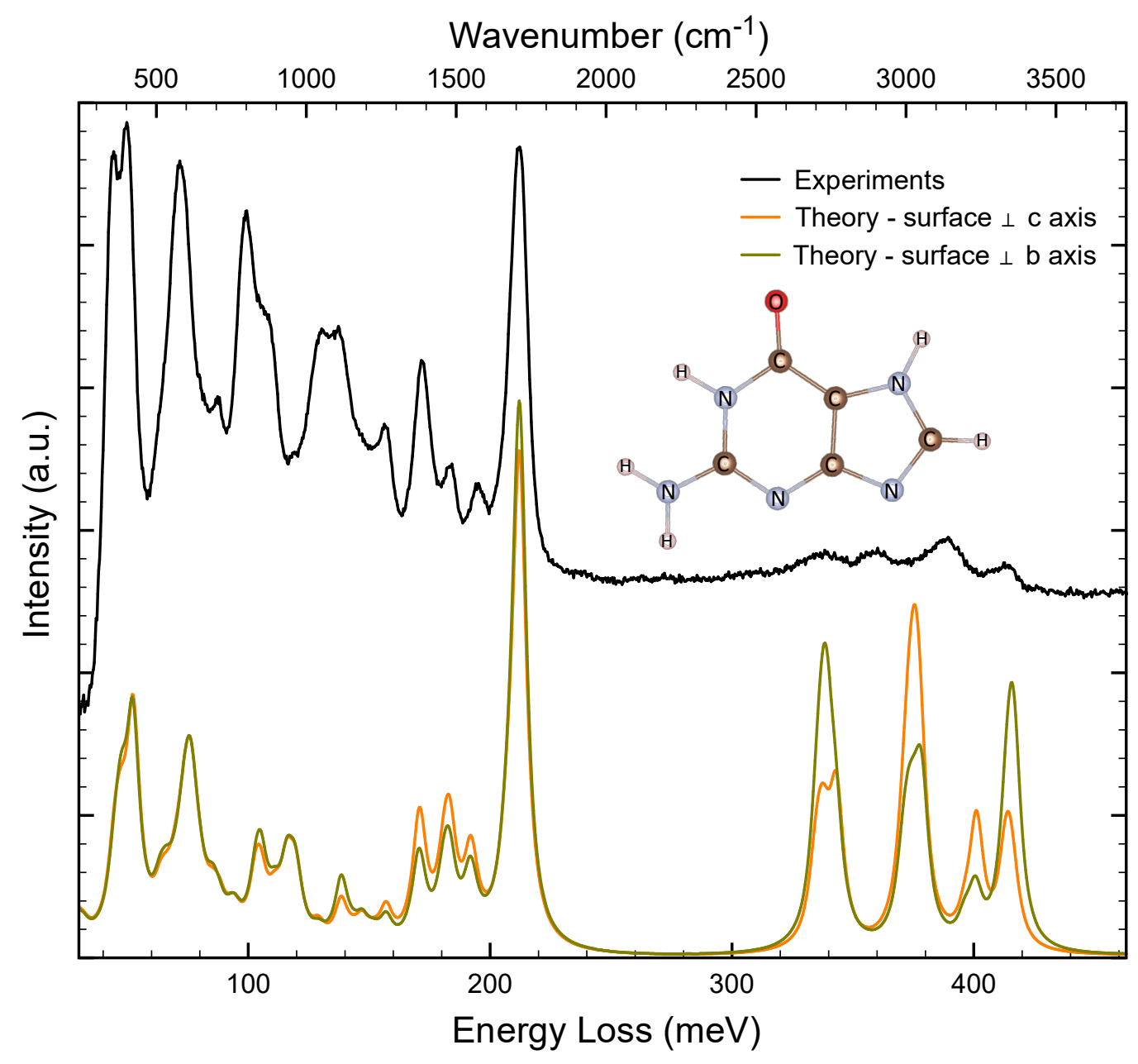

Figure 1. Vibrational EELS spectrum of $\beta$-guanine in near-field geometry: comparison between experiments and theory. Inset: atomic structure of the guanine molecule. 